Case Report

\title{
Bilateral Ganglion Cysts of the Ligamentum Flavum in the Cervical Spine Causing a Progressive Cervical Radiculomyelopathy and Literature Review
}

\author{
Juneki Kim, ${ }^{1}$ Jin-gyu Choi, ${ }^{1}$ and Byung-chul Son ${ }^{1,2}$ \\ ${ }^{1}$ Department of Neurosurgery, Seoul St. Mary's Hospital, College of Medicine, The Catholic University of Korea, \\ Seoul, Republic of Korea \\ ${ }^{2}$ Catholic Neuroscience Institute, College of Medicine, The Catholic University of Korea, Seoul, Republic of Korea \\ Correspondence should be addressed to Byung-chul Son; sbc@catholic.ac.kr
}

Received 29 March 2017; Accepted 3 July 2017; Published 31 July 2017

Academic Editor: Abbass Amirjamshidi

Copyright (C) 2017 Juneki Kim et al. This is an open access article distributed under the Creative Commons Attribution License, which permits unrestricted use, distribution, and reproduction in any medium, provided the original work is properly cited.

\begin{abstract}
Here we report a unique case of bilateral ganglion cysts originating from the ligamentum flavum in the cervical spine. Degenerative cysts of the ligamentum flavum are rare lesions, and most had been reported in the lumbar spine. Its occurrence in the cervical spine is extremely rare: only eight have been reported. A 66-year-old male patient presented with progressive paraparesis, pain, and paresthesia in his bilateral T1 dermatomes that had lasted for three weeks. Magnetic resonance imaging of the cervical spine demonstrated a well-demarcated cystic lesion in the bilateral dorsolateral aspects of the C7/T1 segment and significant compression of the cervical cord. All case reports of ganglion cysts of the cervical ligamentum flavum including the present one showed characteristic symptoms and signs of myelopathy such as paraparesis or quadriparesis associated with varying degrees of paresthesia or pain in the upper extremities. Ganglion cysts of the cervical ligamentum flavum are considered a cause of cervical radiculomyelopathy due to cervical intraspinal cystic lesions. Bilateral occurrence and associated subluxation of the involved cervical segments again support the degenerative pathogenesis of ganglion cysts of the ligamentum flavum in the cervical spine.
\end{abstract}

\section{Introduction}

Ganglion cysts of the ligamentum flavum are uncommon degenerative spinal lesions that are mostly encountered in the lumbar spine [1-6]. Cervical localization is rare and may cause severe myelopathy [7]. These cysts accompany degenerative changes of the spine and can be differentiated from synovial and other degenerative spinal cysts based on location and histopathological features [7]. To our knowledge, only five cases have been reported, and all were associated with myelopathy [7-11]. Here, we report a case of bilateral ganglion cyst of the ligamentum flavum in the cervical spine that presented with gradual paraparesis along with bilateral cervical radicular pain.

\section{Case Presentation}

A 66-year-old male patient, usually in good health, presented progressive paraparesis of three weeks' duration. Three months prior to admission, he had worked hard for four to five hours a day for three months. He developed posterior neck pain and was treated several times with acupuncture. Three weeks earlier, the left leg had lost strength, and his right leg began to flex within a week. Eventually, he could not stand by himself due to the gradual progression of the paraparesis; he also felt paresthesia and pain on his bilateral medial upper arm. No urinary incontinence developed. He was admitted to a hospital and had magnetic resonance imaging (MRI) of the cervical spine and was referred for further evaluation.

Neurologic examination showed paraparesis and ataxia. Decreased motor strength, mainly of the quadriceps and adductors, was noted in his lower extremities, $4 / 5$ on his right side and $3 / 5$ on the left. He had more pronounced deep tendon reflex in his left patellar than in his right one. His upper extremities showed no weakness, but he had pain associated with paresthesia and hypesthesia in bilateral C8 


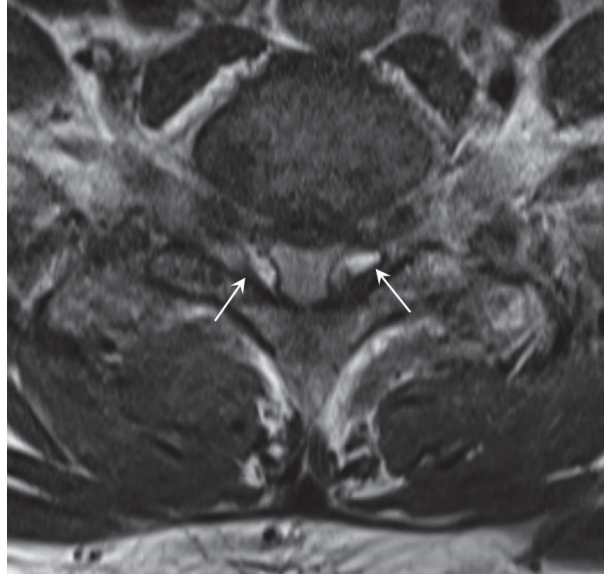

(a) A T2-weighted, axial MRI image showing bilateral ganglion cysts (arrows) of the ligamentum flavum at C7/T1. The rim of the cyst shows low signal intensity connected to the low signal intensity in the ligamentum flavum, and the cyst's contents were heterogenous, with both high and low signals on the left side

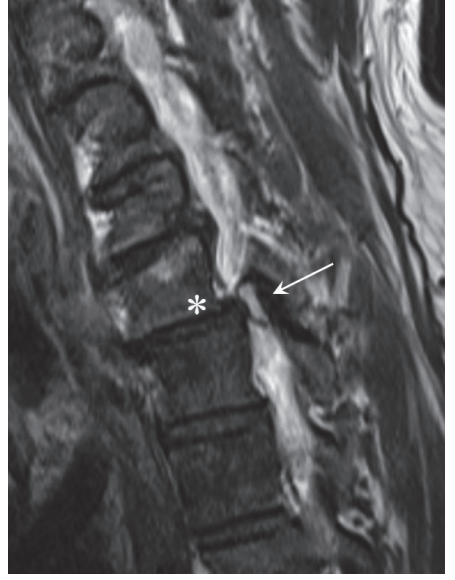

(b) A T2-weighted, sagittal MRI image showing the ganglion cyst (arrow) of the ligamentum flavum at C7/T1. Note the mild anterior subluxation of $\mathrm{C} 7$ to $\mathrm{T} 1$ (asterisk)

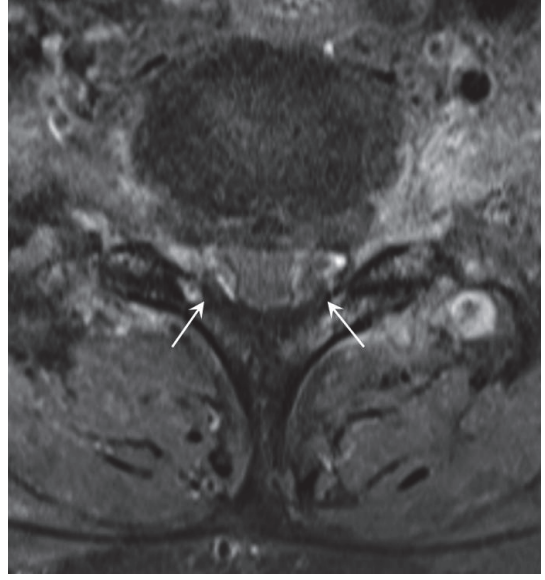

(c) An axial T1-weighted, enhanced MRI image showing variable, contrast enhancement of the cyst wall (arrows)

FIGURE 1: Magnetic resonance imaging (MRI) findings of bilateral ganglion cysts of the ligamentum flavum at C7/T1.

dermatomes. We observed no urinary difficulty. MRI of the cervical spine revealed bilateral extradural cystic masses that compromised the dural sac at the level of C7/T1 (Figure 1). The lesion was isointense in T1-weighted images and showed a hyperintense core with a peripheral hypodense ring in T2-weighted images. The wall of the cyst showed strong enhancement with gadolinium. We observed mild subluxation of C7 over T1 in the sagittal T2-weighted MRI and found prominent degenerative osteoarthritic changes in the bone scan. Considering the progressive neurologic deficits, we planned surgical treatment.

After a laminectomy of C7 and small medial facetectomy of $\mathrm{C} 7 / \mathrm{T} 1$, we removed a hypertrophied ligamentum flavum and exposed the underlying dura. We found a fibrotic, extradural cyst on the internal aspect of the ligamentum flavum (Figure 2) that was embedded within the inner aspect of the ligamentum flavum with no connection with the facet joint or dura mater. When we violated the cyst wall, we found thick, mucous fluid. The cyst wall adhered densely to the underlying, lateral margin of the dura, and we carefully dissected the adhesion under microscopy. After we completely removed the bilateral cysts of the ligamentum flavum and the adjacent hypertrophied ligamentum flavum, we found the dura and the bilateral $\mathrm{C} 8$ root to be decompressed.

The postoperative course was uneventful. Immediately after the operation, the patient's severe pain and paresthesia of the bilateral medial upper arm corresponding to the T1 dermatome were alleviated. Although he experienced immediate functional improvement in both legs, the weakness in both legs improved only slowly over the six months following the operation. There was no weakness or sensory deficit in his arms and legs at the one-year postoperative follow-up and no neck pain or radiological instability.

\section{Discussion}

3.1. Pathogenesis of Ganglion Cysts of the Ligamentum Flavum in the Cervical Spine. Intraspinal degenerative cysts are rare and usually located in the lumbar spine [1-6]. Because the joint capsule is often considered to be the origin of these lesions, they are called juxtafacet cysts to indicate both synovial and ganglion cysts $[12,13]$. Owing to their similar locations and cystic contents, the terms "spinal synovial cyst" and "spinal ganglion cyst" have been used interchangeably [6]. Initially, the suggested difference between synovial and ganglion cysts was that the former often contain clear and serous fluid whereas the latter contain gelatinous, highly viscous fluid [12]. However, differentiation between them is only possible with pathological findings [6]. Synovial cysts are lined with pseudostratified columnar cells, whereas ganglion cysts have no synovial cell lining and no communication with the joint cavity $[6,9,10,13,14]$. In the present case, the cysts did not communicate with the facet joint but were instead imbedded in the ligamentum flavum intraoperatively, and we found no synovial lining on microscopic examination. Therefore, we made the diagnosis of ganglion cyst.

It has been suggested that ganglion cysts are caused by myxoid degeneration and cystic softening of the connective tissue of the joint capsule or tendon sheath as a result of degenerative process or trauma [7]. The pathogenesis of degeneration of the ligamentum flavum is still unclear, but it can be considered in the context of degenerative change [7]. Aging and repeated microtrauma due to spinal motion lead to degenerative changes including loss of elastic fibers, thickening with chondrocyte proliferation and calcifications, and formation of collagen fibers [6]. Loss of elasticity predisposes the ligamentum flavum to mechanical stress injury, resulting in scar remodeling and ganglion cyst formation [6]. The 


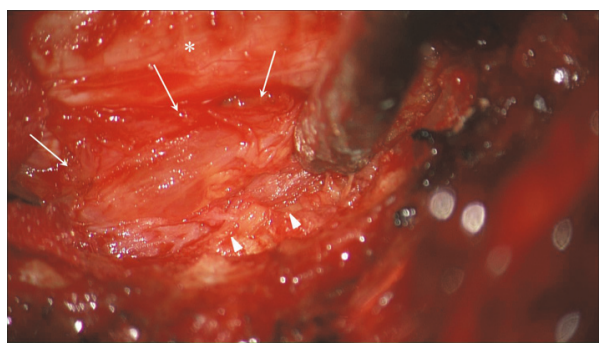

(a) An intraoperative photograph showing the outer wall of ganglion cyst (arrows) at the right side of C7/T1. The ligamentum flavum was bulged with the cyst, and the lateral margin of the dura (asterisk) was compressed. The cut edge of the facet (arrowheads)

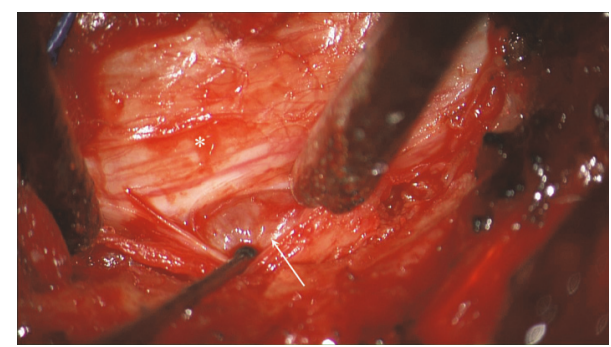

(b) After the outer wall of the ligamentum flavum cyst was cleared, a degenerated cystic portion was found (arrow). The underlying dura (asterisk) was dissected and decompressed

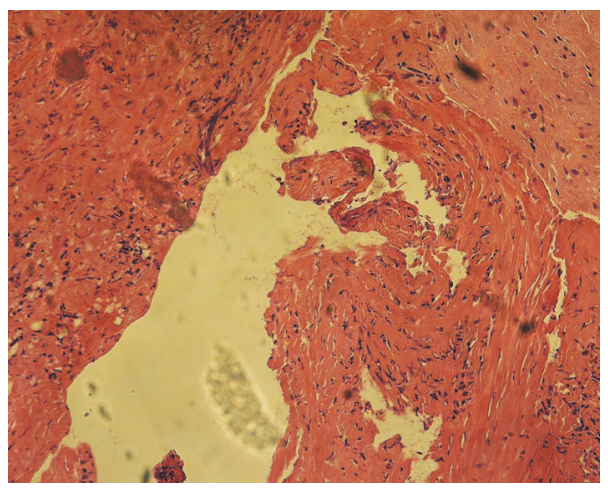

(c) A histologic specimen showing the absence of synovial cell lining of the inner cystic wall $(\times 40, \mathrm{H} \& \mathrm{E}$ stain)

FIGURE 2: Intraoperative photograph showing the ganglion cyst of the ligamentum flavum.

current case is unique because of the bilateral occurrence of the ligamentum flavum ganglion cyst and associated mild subluxation. We think that bilateral occurrence and associated subluxation further support the pathogenesis of degenerative changes involving the ligamentum flavum. Indeed, in the literature, all of the case reports involved people over age 60 (Table 1).

\subsection{Symptoms, Diagnosis, and Treatment of Ganglion Cysts} in the Cervical Ligamentum Flavum. Symptoms and signs of myelopathy with or without cervical radiculopathy association are the most common presenting symptoms in symptomatic ganglion cysts of the ligamentum flavum in the cervical spine. In our review of the literature regarding theses cysts, six of seven reported cases (86\%), including the current case, showed paraparesis or quadriparesis owing to their location within the narrow cervical spinal canal. Most symptoms and signs of myelopathy are gradual and insidious (Table 1). However, an occurrence of sudden Brown-Sequard syndrome within three hours due to a ganglion cyst of the cervical ligamentum flavum has been reported [10]. Although the symptomatic cervical ligamentum flavum ganglion cysts in the literature have been small (8 to $15 \mathrm{~mm}$ in diameter), all reported cases showed characteristic symptoms and signs of myelopathy: gait disturbance and paraparesis [15-17].
MRI is the imaging study of choice in the diagnosis of ganglion cysts in the cervical spine, although histopathologic examination is needed for definitive diagnosis [7]. The MRI findings of the ganglion cysts are characteristic; the cyst contents are hypointense on T1-weighted images and hyperintense on T2-weighted images $[6,7,9,10,14]$. The peripheral rims of the cysts are hypointense on T2-weighted images with gadolinium enhancement. The treatment for symptomatic ganglion cysts of the ligamentum flavum in the cervical spine is surgery. All reported cases of ganglion cervical spine cysts caused serious neurologic deficits, and surgical treatment universally resulted in neurologic improvement.

Surgical excision of ganglion cysts of the cervical spine with posterior laminectomy appears to be a straightforward for decompressing the spinal cord, removing the ganglion cysts, addressing the connection to the facet joint, and taking histologic specimens for definitive diagnosis. All case reports regarding symptomatic ganglion cysts of the cervical ligamentum flavum adopted surgical excision via laminectomy, and the prognosis of surgical treatment is favorable. Although some degree of adhesion between the dura and the ganglion cyst was always mentioned, no surgical morbidity or neurologic compromise was reported. Gradual recovery of the symptoms and signs of radiculomyelopathy appear to occur within 6 to 12 months postoperatively. 


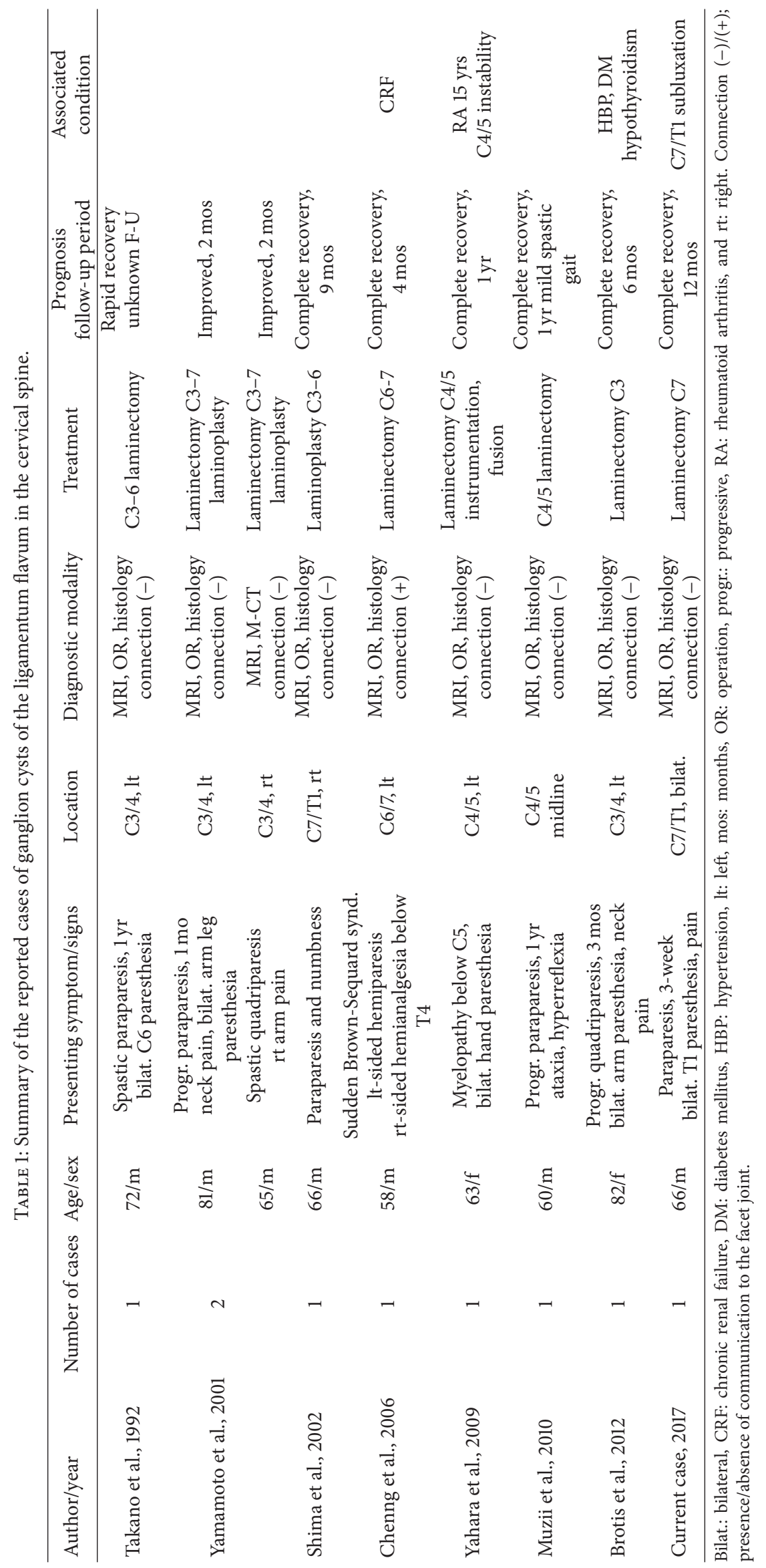




\section{Conclusions}

We here report a very rare case of bilateral ganglion cysts of the cervical ligamentum flavum that presented with progressive myelopathy and radiculopathy. The characteristic bilateral occurrence and associated cervical subluxation at the involved segment supported the degenerative pathophysiology in the cyst.

\section{Conflicts of Interest}

The authors declare no conflicts of interest regarding this manuscript.

\section{References}

[1] A. F. Abdullah, R. W. Chambers, and D. P. Daut, "Lumbar nerve root compression by synovial cysts of the ligamentum flavum. Report of four cases," Journal of Neurosurgery, vol. 60, no. 3, pp. 617-620, 1984.

[2] J. K. Baker and G. W. Hanson, "Cyst of the ligamentum flavum," Spine, vol. 19, no. 9, pp. 1092-1094, 1994.

[3] C. B. Bärlocher and R. W. Seiler, "Vertebral erosion and a ligamentum flavum cyst," Journal of Neurosurgery: Spine, vol. 93, no. 2, pp. 335-335, 2000.

[4] J. Haase, "Extradural cyst of ligamentum flavum 14 - A case," Acta Orthopaedica, vol. 43, no. 1, pp. 32-38, 1972.

[5] H. Terada, Y. Yokoyama, N. Kamata, T. Hozumi, and T. Kondo, "Cyst of the ligamentum flavum," Neuroradiology, vol. 43, no. 1, pp. 49-51, 2001.

[6] A. Yamamoto, I. Nishiura, H. Handa, and A. Kondo, "Ganglion cyst in the ligamentum flavum of the cervical spine causing myelopathy: report of two cases," Surgical Neurology, vol. 56, no. 6, pp. 390-395, 2001.

[7] V. F. Muzii, P. Tanganelli, G. Signori, and A. Zalaffi, "Ganglion cyst of the ligamentum flavum: a rare cause of cervical spinal cord compression. A case report," Journal of Neurology, Neurosurgery and Psychiatry, vol. 81, no. 8, pp. 940-941, 2010.

[8] A. G. Brotis, E. Z. Kapsalaki, E. K. Papadopoulos, and K. N. Fountas, "A cervical ligamentum flavum cyst in an 82-year-old woman presenting with spinal cord compression: a case report and review of the literature," Journal of Medical Case Reports, vol. 6, article no. 92, 2012.

[9] Y. Takano, T. Homma, H. Okumura, and H. E. Takahashi, "Ganglion cyst occurring in the ligamentum flavum of the cervical spine: a case report," Spine, vol. 17, no. 12, pp. 1531-1533, 1992.

[10] W. Y. Chenng, C. C. Shen, and M. C. Wen, "Ganglion cyst of the cervical spine representing with Brown-Sequard syndrome," Journal of Clinical Neuroscience, vol. 95, supplement 1, pp. 193$142,2006$.

[11] L. F. Chen, C. C. Lui, M. H. Cheng, and J. W. Lin, "Ganglion cyst in the ligamentum flavum of the cervicothoracic junction," Journal of the Formosan Medical Association, vol. 95, pp. 490492, 1996.

[12] C. C. Kao, S. S. Winkler, and J. H. Turner, "Synovial cyst of spinal facet. Case report," Journal of Neurosurgery, vol. 41, no. 3, pp. 372-376, 1974.

[13] O. Hatem, G. Bedou, C. Négre, J. L. Bertrand, and J. Camo, "Intraspinal cervical degenerative cyst. report of three cases," Journal of Neurosurgery, vol. 95, no. 1, pp. 139-142, 2001.
[14] M. A. Stoodley, N. R. Jones, and G. Scott, "Cervical and thoracic juxtafacet cysts causing neurologic deficits," Spine, vol. 25, no. 8, pp. 970-973, 2000.

[15] P. Fransen, G. P. Pizzolato, P. Otten, A. Reverdin, R. Lagier, and N. De Tribolet, "Synovial cyst and degeneration of the transverse ligament: an unusual cause of high cervical myelopathy. case report," Journal of Neurosurgery, vol. 86, no. 6, pp. 10271030, 1997.

[16] Y. Shima, S. L. G. Rothman, K. Yasura, and S. Takahashi, “Degenerative intraspinal cyst of the cervical spine: case report and literature review.", Spine, vol. 27, no. 1, pp. E18-22, 2002.

[17] Y. Yahara, Y. Kawaguchi, S. Seki, Y. Abe, T. Oya, and T. Kimura, "Ligamentum flavum cyst of the cervical spine associated with rheumatoid arthritis," Journal of Orthopaedic Science, vol. 14, no. 2, pp. 215-218, 2009. 


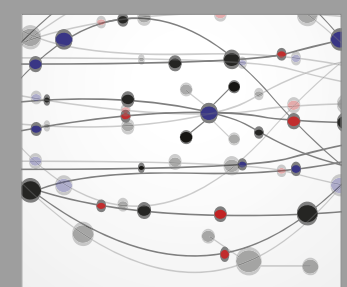

The Scientific World Journal
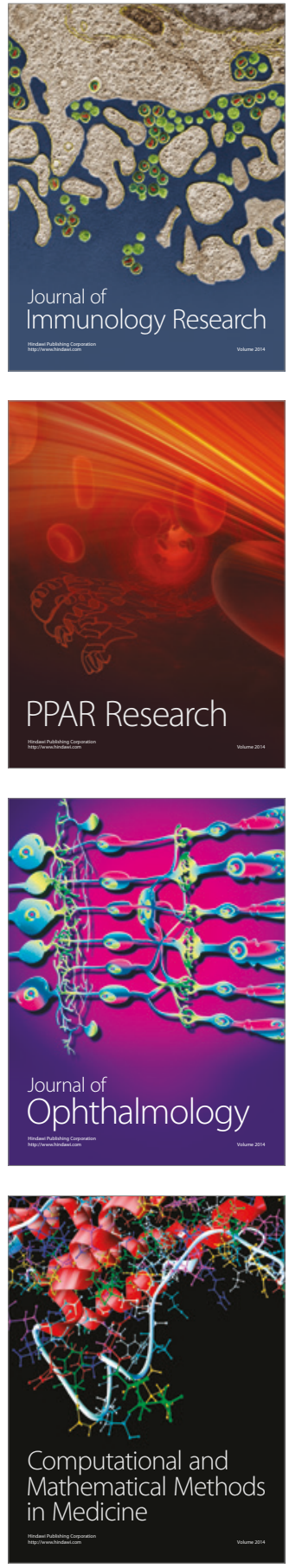

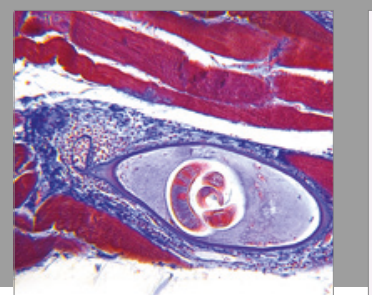

Gastroenterology Research and Practice
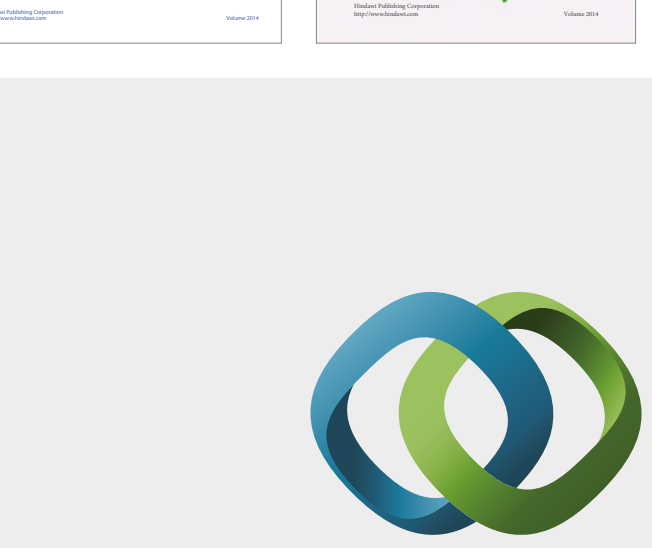

\section{Hindawi}

Submit your manuscripts at

https://www.hindawi.com
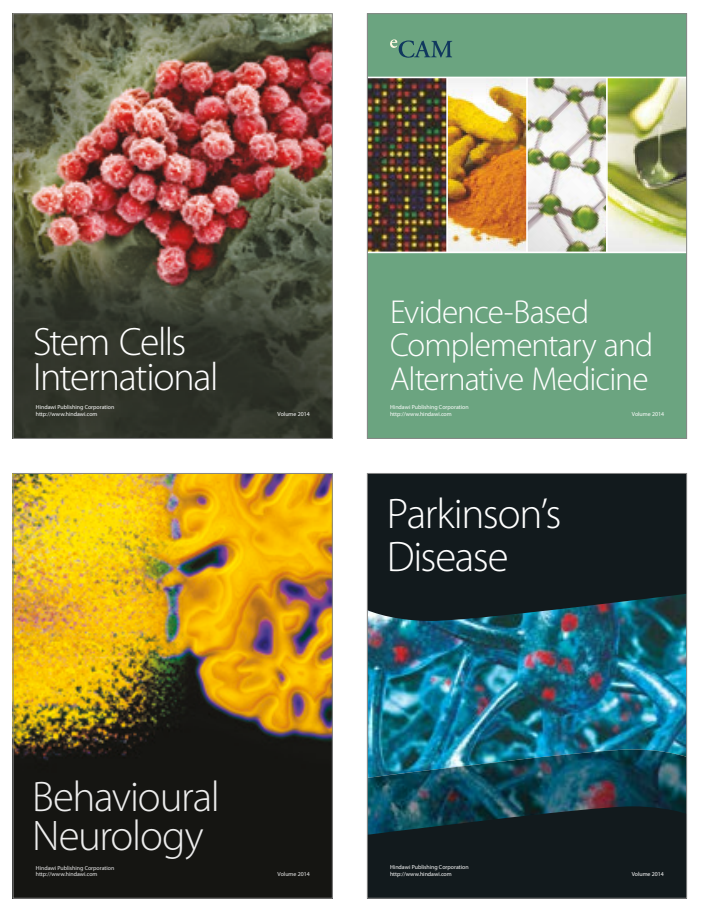
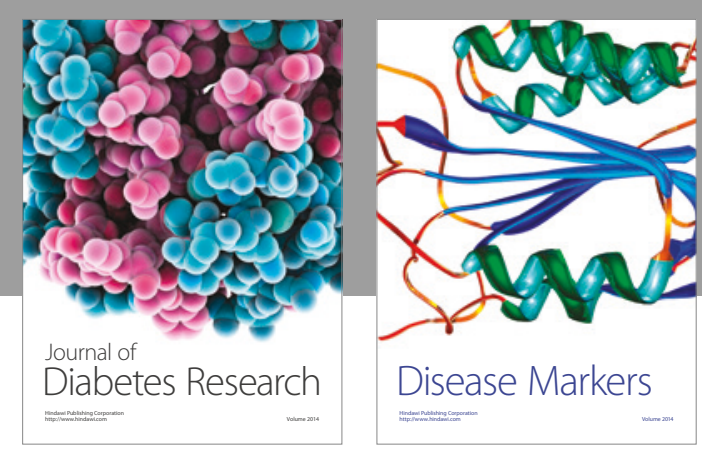

Disease Markers
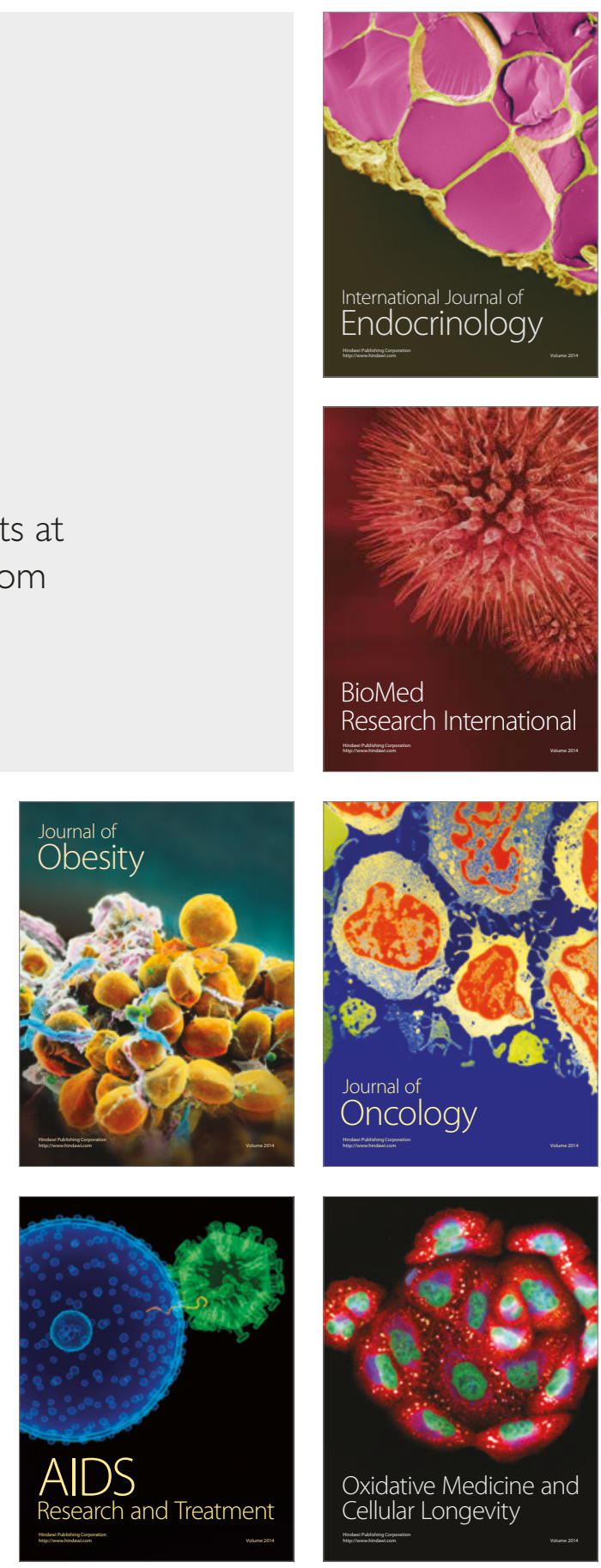